\title{
Lifetime History of PreVEntion IN EUROPEAN COUNTRIES: the Case of Dental Check-ups
}

Brigitte Santos-Eggimann, Sarah Cornaz, Jacques Spagnoli 


\title{
21 Lifetime History of Prevention in European Countries: the Case of Dental Check-ups
}

\author{
Brigitte Santos-Eggimann, Sarah Cornaz and Jacques Spagnoli
}

\subsection{Access to dental care: an indicator of performance of health care systems}

European countries differ in various aspects of their health care systems, including the level of public investments and the importance given to prevention in national health policies. However, little is known regarding the association between the dissemination of preventive practices in populations included in the SHARELIFE project and public policies in health domain.

Access to care is a major characteristic for the performance of health care systems. Its assessment includes measurements of the reported use for a range of ambulatory and hospital services considered as an indicator of realized access. Some aspects of health care are particularly sensitive to the risk of inequality in access. It is the case of dental care. Indicators of access to dental care are part of recent WHO and OECD reports on health and care (WHO, 2010; OECD, 2009); they show a strong relationship with individuals' socio-economic circumstances (De Looper and Lafortune, 2009; Van Doorslaer and Masseria, 2004). Previous results from an analysis of SHARE baseline data indicated that, in participating European countries, visits to the dentist were unrelated to gender but increased with the level of education (Santos-Eggimann et al., 2005). Preservation of the mastication capacity is crucial to avoid denutrition and its consequences on health and function in older age. While in most countries European recommendations are issued for yearly dental check-ups, dental care remains costly, it is mainly offered by professionals active in the private sector and its coverage by social health insurances varies between countries from no one in Spain to complete in Austria (OECD, 2009).

This chapter has the first objective to describe holes in the lifetime use of routine preventive dental controls in the SHARELIFE population, by country. The second aim is to confront differences in underuse of dental check-ups observed across European countries with contextual indicators that describe national human resources and reimbursement policy regarding dental care. 


\subsection{Data on individuals and health systems}

SHARELIFE, the third wave of SHARE data collection, included original retrospective questions on individual lifetime use of dental care. They concentrated on dental check-ups and care regularly performed from childhood to the time of interview. Information was collected on the age of respondents when starting to visit a dentist regularly, the average frequency of dental visits, periods of interruption and related age category of respondents, as well as reasons for not having dental check-ups and care regularly over the life course. For each respondent, we defined holes in the lifetime use of routine preventive dental controls as periods in which dentists were either not regularly visited, or visited less frequently than every two years. These periods were characterized by the age of respondents: during childhood (0-15 years), youth (16-25 years), young adulthood (26-40 years), adulthood (41-55 years), middle age (56-65 years) and older age (66-80 years).

Analyses of SHARELIFE individual data were performed on respondents aged 52 to 80 years from 13 countries participating in SHARELIFE, taking account the complete design information available from SHARELIFE and Share wave 2 to compute a correct estimate of variance. In order to take account of cohort effects, analyses were conducted for respondents aged 52 to 65 years and 66 to 80 years separately.

Results are displayed in 4 geographic regions: Northern (Sweden SE, Denmark DK, Netherlands NL), Continental (Belgium BE, Germany DE, Austria AT, France FR, Switzerland CH), Southern (Spain ES, Italy IT, Greece GR) and Eastern (Czech Republic CZ, Poland PL) countries.

A total of 21281 respondents born between 1928 and 1956 were included in analyses. The sample size ranged from 711 in AT to 2286 in GR. Respondents were categorized into two age classes, 52-65 years $(57.0 \%)$ and $66-80$ years $(43.0 \%)$. The proportion of women was $55.7 \%$ in the first and $52.9 \%$ in the second age category.

Context variables describe national manpower (density of dentists: number of dentists in practice / 1'000 inhabitants) from 1960 to 2006, according to 2008 OECD Health data, and the coverage of dental care (out-of-pocket dental expenditure as a percentage of total dental expenditure) in 2006, according to OECD indicators (OECD 2009). Additional information on health policy for selected countries was collected from WHO European observatory of health systems reports (WHO, 2010).

\subsection{Lifetime underuse of dental care in European countries}

Both the level and the pattern of the lifetime underuse of dental care of middle aged individuals, as measured from self-reported information, varies widely be- 
tween European regions. Figure 21.1 shows that, over the lifetime, underuse of dental care in subjects currently aged 52-65 years was at the lowest level and stable in all three Northern countries. Along the whole life course, a slightly higher (although not significantly) proportion of underuse is reported in NL as compared to DK and SE, except in the childhood period. In all three countries, underuse does not exceed $17 \%$ at all ages, with very close proportions observed in DK and SE.

Countries of the Continental region of Europe share a common pattern of lifetime experience: BE, DE, AT, FR and $\mathrm{CH}$ all experience a decline in underuse of dental care over the life course. However, the level of underuse varies substantially between these countries. It is the highest in BE and FR, where proportions decline from $62 \%$ in childhood to $43 \%$ at age 56-65 years, with a slightly higher level in BE than in FR. Underuse decreases during the life course from $44 \%$ in childhood to $33 \%$ at age 56-65 years in AT, and from $41 \%$ to $23 \%$ in DE. It is the lowest and characterized by the smallest decline over the life course in $\mathrm{CH}$ (from $23 \%$ to $17 \%$ ). Figure 21.1 also shows that the ranking of countries in Continental Europe is maintained all over the life course, with Belgian respondents experiencing the highest level of underuse from their childhood to their middle age, and Swiss respondents reporting the lowest level of underuse during their whole life.

Like in Continental Europe, countries in the Southern region display a decline in underuse of dental care with age. However, their level is very high, with proportions of underuse ranging between $82 \%$ and $88 \%$ in childhood, and between $62 \%$ and $71 \%$ at age 56-65 years. GR experience a particularly high proportion of underuse of dental care in adulthood, while ES and IT share a similar experience of underuse along the whole life course.

In Eastern Europe, $\mathrm{CZ}$ and PL display a similar pattern of underuse unaffected by age. The level is low (between $8 \%$ and $10 \%$ ) in CZ, where it compares with the experience of Northern countries. By contrast, it is high in PL (between 58\% and $63 \%$ ), where it compares with Southern countries in adulthood.

Figure 21.1: Proportions of SHARELIFE respondents aged 52 to 65 years in 2008 who underused dental care at given ages 

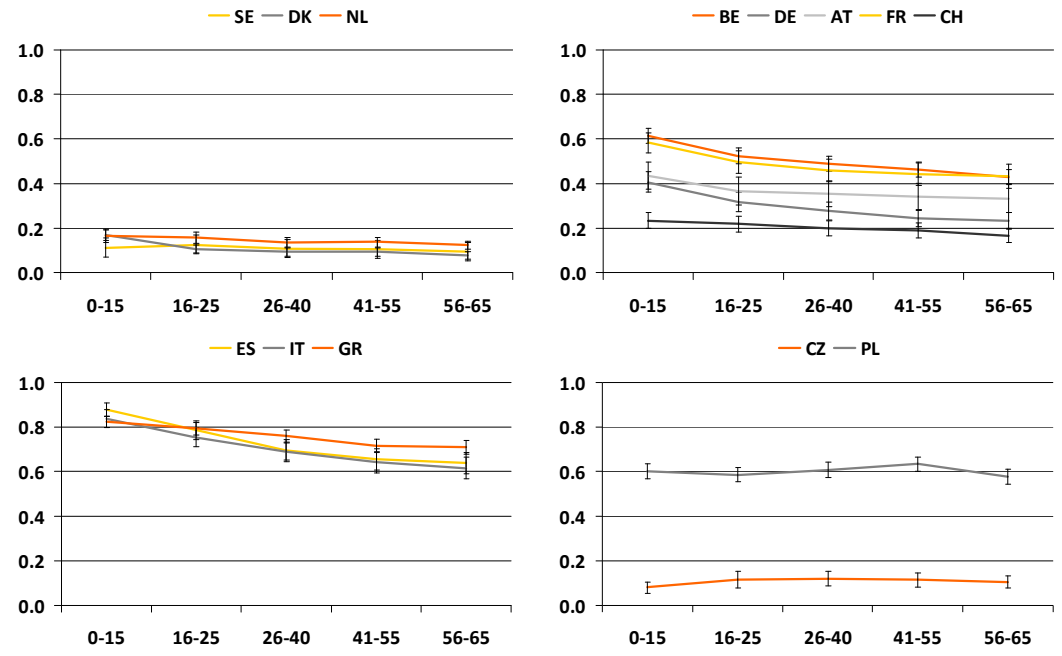

The life course experience of dental care in European populations seems to have improved over time in all countries participating in SHARE. A comparison between Figures 21.1 and 21.2 suggests a cohort effect: in all countries except SE, the level of underuse reported by individuals aged 66 to 80 years (Figure 21.2) is higher during the whole life course, than the level reported by the 52 to 65 years old (Figure 21.1).

In the Northern region, differences between countries are larger than in the younger age category, and Figure 21.2 displays an age effect that was not observed in Figure 21.1: underuse of dental care reported by individuals aged 66 to 80 years was higher in their childhood and youth than in their adulthood and in older age. NL shows the highest level of underuse, ranging between $45 \%$ in childhood and around 30\% from age 16-25 to age 76-80 years, and SE the lowest level (from $19 \%$ to $10 \%$ ).

In the Continental region, the ranking of countries is similar in Figures 21.1 and 21.2 except for DE. Underuse of dental care declines from $79 \%$ in childhood to $59 \%$ at age 76 to 80 years in $\mathrm{BE}$, and from $48 \%$ to $34 \%$ in $\mathrm{CH}$. However, the lifetime evolution of underuse for 66 to 80 year old individuals in DE is characterized by a high proportion of underuse in childhood (59\%, rank $3 / 5)$ to a low proportion in the adulthood and older age (25\% at age 76 to 80 years, rank 5/5).

A high level of underuse over the life course is registered in Southern countries for respondents aged 66 to 80 . The order of countries is the same as the order observed for the younger age category of respondents; differences between countries, however, are larger and increasing over the life course. The level of underuse declines slightly from $94 \%$ in childhood to $85 \%$ at age 76 to 80 years in GR. The slope is steeper in ES (from 94\% to 76\%) and in IT (from 90\% to 68\%).

In Eastern Europe, the life course decline of underuse reported by individuals aged 66 to 80 is moderate, and the level of this indicator is much lower in $\mathrm{CZ}$ 
(from $25 \%$ in childhood to $18 \%$ at age 76 to 80 years) than in PL (from $83 \%$ to $72 \%)$.

Figure 21.2: Proportions of SHARELIFE respondents aged 66 to 80 years in 2008 who underused dental care at given ages
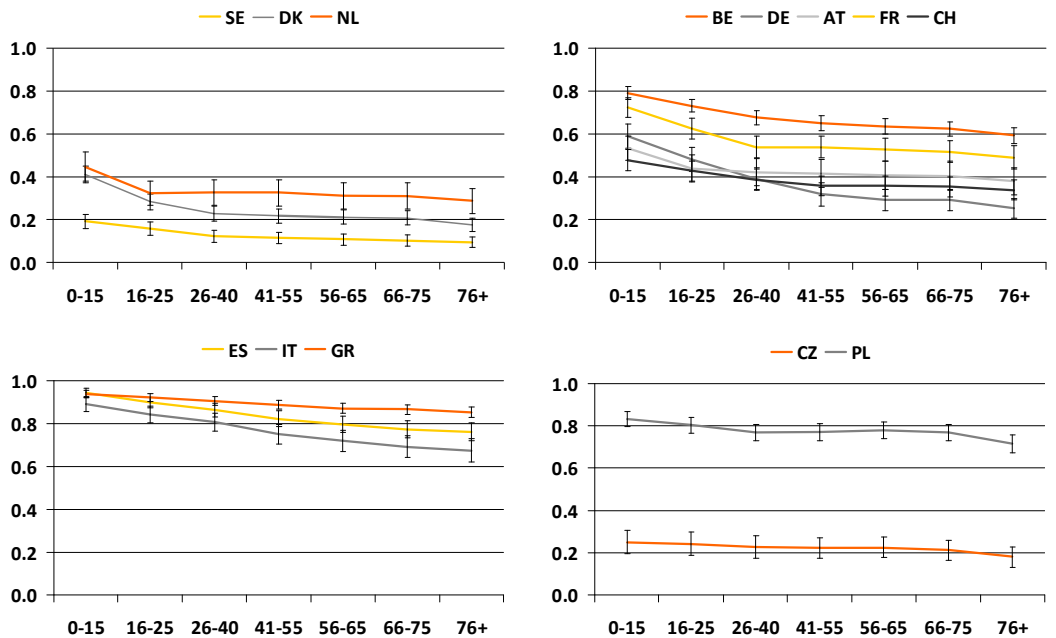

\subsection{Elements of health policy regarding dental care in European countries}

In most countries participating in SHARELIFE, there is no clear evidence of a relationship between the level of underuse of dental check-ups and care and the density of dentists as shown in Figure 21.3. While the level of underuse reported by respondents aged 52 to 65 is very close over their whole life course in the three Northern countries, a sizable difference in dentists' density is observed between NL (low density) and SE (high density), with DK in an intermediate position. This ranking, however, reflects the order of countries observed in the older age group, with a lower level of underuse over the life course in SE, a higher level in NL, and an intermediate level in DK. Differences today are smaller, with a level at 0.5 dentists / 1'000 inhabitants in NL and 0.8 in SE and DK. In these Northern countries, the current proportion of dental expenditure paid out-of-pocket is estimated at $63 \%$ in SE and at $69 \%$ in DK (no data available in NL).

Countries in the Continental region have less difference in dentists' density. DE has the highest density over the whole 1960 to 2006 period. BE was characterized by a low density in 1965-1969 and a continuous increase in the following 40 years; it ranks as high as DE in the most recent year (0.8). FR also increased its 
density in the past 30 years and ranks slightly below DE and BE in 2005 (0.7). AT and $\mathrm{CH}$ are in the lowest range among these countries, with a quite stable density that compares to the density of NL today (0.5). Despite their increasing dentists' density over time, and despite a proportion of dental expenditure paid out-ofpocket in the low range (BE 34\% and FR 28\%), these two countries have the highest level of underuse of dental care in the Continental region. By contrast, DE, cumulating a high dentists' density over the last 45 years and the lowest out-ofpocket share of dental care expenditure, has a moderate level of underuse. The Swiss situation, where dentists' density is in the middle range and dental care expenditures are only marginally financed by the community (out-of-pocket share 91\%), is particular, with a favorable position regarding underuse of dental care.

Among Southern countries, ES was characterized by a particularly low density of dentists from 1960 to the middle of the years 1980. Density then increased up to 0.5 in 2006. This level compares with IT in the recent years (no data available before 1993). By contrast, the dentists' density in GR was at 0.4 in 1960 and increased to 1.2 in 2005 . The very high proportion of the population experiencing underuse of dental care along the whole life course in ES may be explained not only by a low density of professionals, but also by the fact that dental care is almost exclusively paid out-of-pocket $(97 \%)$ in this country. Information regarding the share of dental care expenditure paid out of pocket in GR and IT is not available in the OECD database.

In the Eastern region, the evolution of dentists' density differs between $\mathrm{CZ}$, where it increased from a low 0.2 dentists / 1'000 inhabitants in 1970 to 0.7 in 2006, and PL, where dentists' density increased from 0.3 in 1960 to 0.5 in 19771991, and then declined to reach 0.3 in the end of the 1990 years. The share of out-of-pocket financing of dental care is lower in CZ (30\%) than in PL (69\%), which may explain the much higher level of underuse of dental care over the life course in PL.

Figure 21.3: Number of dentists in practice / 1'000 inhabitants) from 1960 to 2006 or last year available, by country (source: OECD Health data 2008) 

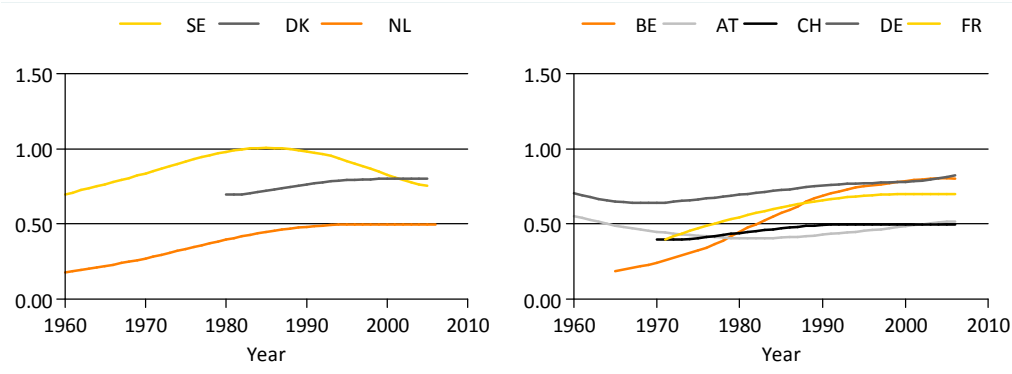

$\longrightarrow$ ES $-\mathrm{IT} \longrightarrow \mathrm{GR}$

$-\mathrm{CZ}-\mathrm{PL}$
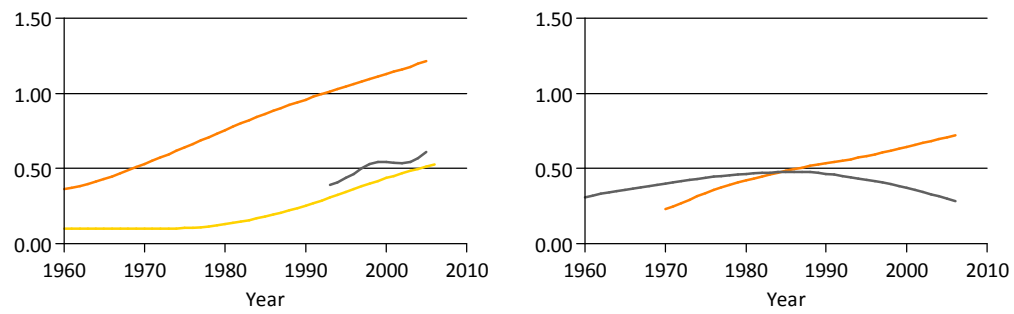

\subsection{Reasons for missing routine dental controls in European countries}

SHARE participants reporting holes in their lifetime use of routine preventive dental controls were also asked about causes. In all countries, a majority of respondents mentioned that they did not perceive a need, it was not usual to have dental check-ups, or information was lacking.

Figure 21.4: Proportion of respondents reporting holes in their lifetime use of routine preventive dental controls who mentioned that that they did not perceive a need, it was not usual to have dental check-ups, or information was lacking. 


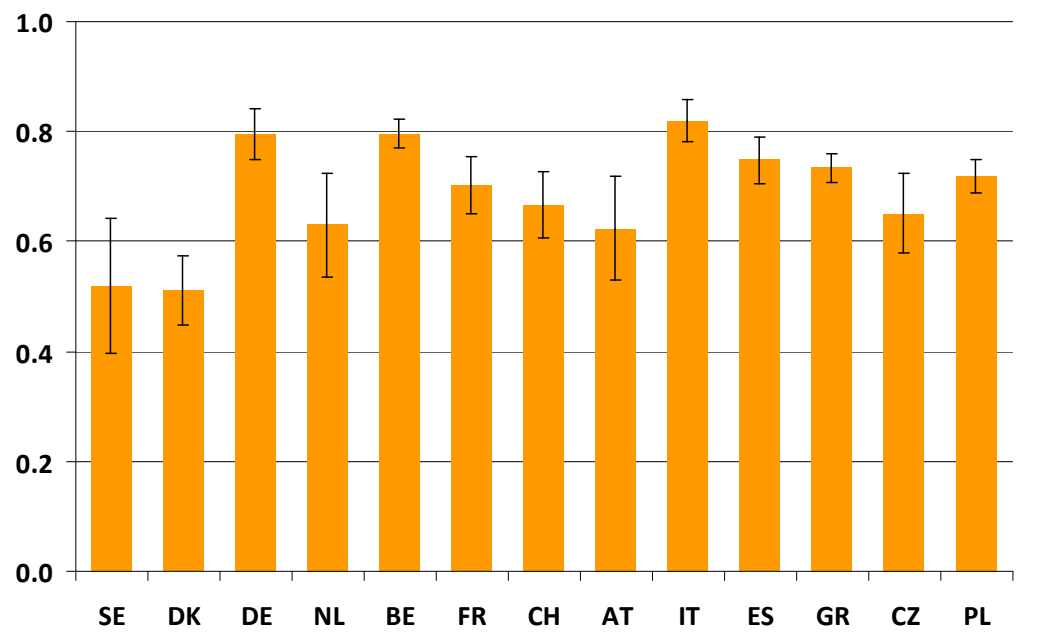

The proportion mentioning that they had no place to receive this type to care near home did not exceed 5\%, except in GR (18\%) and in PL (12\%). Overall, financial reasons (cost of care or lack of insurance coverage) were mentioned in $16 \%$, but the proportion exceeded $20 \%$ in $\mathrm{CH}(30 \%)$, ES $(29 \%)$, SE $(28 \%)$, DK (24\%) and GR $(21 \%)$.

\subsection{Conclusions}

Underuse of dental care over the life course compromises health in old age and should be limited to improve the quality of life. SHARELIFE data indicate that even in countries characterized by the most favorable conditions of professional manpower and care reimbursement, and using a very conservative estimate of underuse of dental care (periodicity of controls at least every two years was the cutoff used in this analysis, while most countries recommend yearly visits to the dentist), underuse of dental care concerns at least $10 \%$ of the population aged 52 to 80 years.

Large variations were observed between European regions and countries, with levels of underuse reaching more than $85 \%$ along the whole life course in some countries. In most cases, high levels of underuse can be explained by a dentists' density lower than 0.5 / 1'000 inhabitants and / or by a large proportion of dental care bills paid out-of-pocket. Intriguing situations, like $\mathrm{CH}$ where the level of underuse is moderate and the proportion of dental care expenditure financed out-ofpocket is very high, may have roots in a sufficient dentists' density conjugated with a high level of income. In GR, the high level of underuse of dental care de- 
spite a very high density of dentists may be related to the high cost of this type of care (Siskou et al, 2009) or to variations in density by region (e.g. Athens 1.66 / 1'000 inhabitants, Ionian Islands 0.57 / 1'000 inhabitants in 2001, according to data provided by the Hellenic Statistical Authority (EL.STAT.)). Further investigations should be dedicated to gain a better understanding of the specific situation in countries or regions, and to integrate the potential multiple causes of underuse of dental care in Europe.

\section{References}

De Looper, M., Lafortune, G. 9.3.2009. Measuring disparities in health status and in access and use of health care in OECD countries. OECD health working papers 43.

Hellenic Statistical Authority (EL.STAT.), www.statistics.gr (accessed April 2010).

OECD Health data 2008: Statistics and indicators for 30 countries. 26.6.2008. Paris.

OECD Health at a glance 2009 - OECD Indicators. 8.12.2009. Paris.

Santos-Eggimann, B., Junod, J., Cornaz, S. 2005. Health services utilization in older Europeans. First results from the Survey of Health, Ageing and Retirement in Europe. Mannheim, pp. 133-140.

Siskou, O., Kaitelidou, D., Economou, C., Kostagiolas, P., Liaropoulos, L. 2009. Private expenditure and the role of private health insurance in Greece: Status quo and future trends. European Journal of Health Economics, Vol. 10, pp. 467-474.

Van Doorslaer, E., Masseria, C. 11.5.2004. Income-related inequality in the use of medical care in 21 OECD countries. OECD health working papers 14. Paris.

WHO European Observatory on Health Systems and Policies www.euro.who.int/observatory (accessed 4.3.2010) 